\title{
Flow Field Simulation between Angle Vane and Cylinder
}

\author{
${ }^{1}$ Rafi M. Qasim, ${ }^{2}$ Tahseen A. Jabbar, ${ }^{3}$ Ihsan A. Abdulhussein \\ \{rafi.mohammed@stu.edu.iq ${ }^{1}$, tahseen.ali@stu.edu.iq ${ }^{2}$,drengihssan@stu.edu.iq ${ }^{3}$ \} \\ ${ }^{1,2}$ Fuel and Energy Department, Basrah Engineering Technical College, Southern Technical University, \\ Basra, Iraq \\ ${ }^{3}$ Basra Engineering Technical College, Southern Technical University, Basra, Iraq
}

\begin{abstract}
Water flow around cylinder represents a noticeable problem in fluid mechanics. The computational fluid dynamic is employed to study numerically the turbulent flow field around a cylinder without vane and with vane respectively. The vane configuration and direction are considered in the numerical study. The analysis is done in two dimensions by using ANSYS software. The finite volume method is used to model and simulate the cases study. The numerical study concentrates on strain rate, stream function, swirling, vorticity, velocity in the flow direction, radial velocity, pressure distribution, pressure coefficient and drag coefficient. It is recognizing that the vane changes the flow field hydraulic response. It is found from the result that the vane more reduces the value of the drag coefficient as compared with the value of the drag coefficient when the cylinder is alone. The stream function reveal how much the fluid suffer from deformation while the strain rate reveal how much t5he flow velocity change with distance. Also, vorticity illustrate a dramatic hydraulic response especially in the case of the vane existence. The study shows that the vane has direct effect on the flow separation zone and dissipation the turbulent intensity before it passes the cylinder.
\end{abstract}

Keywords: Cylinder, Flow Field, Fluid-Structure Interaction, Vane.

\section{Introduction}

One of the most dominant problems of flow-cylinder structure interaction is the turbulent flow around a circular cylinder which is placed vertically or in inclined position inside the flow respectively. Such a pier radically changes the flow around it. It is very important to study the flow field around a cylinder under different conditions like placing an obstacle, for example vane, before the cylinder at upstream to reduce the turbulence and separation zone of the flow. Many papers dealt with the problem of flow around a cylinder like flow around bridge pier, pile and pile groups. Sowoud et al. [1] investigated the turbulence model applicability in the engineering practice for the case of subcritical flow and examined the variation of Reynolds number on the characteristics of the flow. ANSYS FLUENT (19.1) is used to perform the numerical simulation in two dimensions. Majtaba Daneshi [2] did a numerical investigation for the flow characteristics at the downstream of a circular cylinder, ANSYS Fluent and the two-dimensional finite volume 
method is used to perform the numerical analysis. Here, the characteristics are investigated under the following conditions: laminar and turbulent unsteady incompressible flow. The characteristics include drag force, pressure distribution and velocity fields. Also, the effect of Reynolds number on the flow parameter is investigated. Kartik Chandra Bhagat et al. [3] carried out a numerical and an experimental work to obtain the pressure coefficient and drag force for various aspect ratio $(\mathrm{L} / \mathrm{D})$. The numerical analysis is performed by adopting ANSYS Fluent 14.5 to investigate the pressure distribution around a circular cylinder and point of the flow separation. Yuce and Kareem [4] made a numerical investigation to investigate the flow field around the cylinders with the same characteristic length under the same flow conditions with the ranging from the laminar flow to the turbulent flow. The simulation is performed in two dimensions. The flow field was found to be affected by the shape of the cylinder, wake downstream of the square cylinder was found to be much more turbulent than that of the circular one. Also, the increased in Reynolds number will reflect on the turbulence of the wake flow and its length at the downstream of the cylinders. Wei and Aode [5] used the method of large-eddy simulation to investigate numerically the scour and the turbulent flow field around a circular pier. From the result they found decreasing in shear stress with increase in the scour depth, also a gradual increase occurred in the gradient of vertical pressure and turbulent intensity. Davis and Moore [6] investigated numerically the flow which pass a rectangular cylinder. Dependent on the mean inlet flow velocity and the cylinder height, they mentioned the forces which are acting on the cylinder and the structure of the wake with different aspect ratio and the angles of the attack at two values of the Reynolds number. Oudheusden et al. [7] carried out an experimental to investigate the vorticity near a square cylinder by adopting PIV technology, where the Reynolds which is adopted in the experiment work has the values of 4000, 10000 and 20000. Gera et al. [8] investigated two dimensional unsteady flows by using the finite element method around a cylinder with quadrilateral shape, and Reynolds number range from 50 to 250 . Bai and $\mathrm{Li}$ [9] used a computational fluid dynamic technique to simulate unsteady flow around circular cylinder in two dimensions with Reynolds number equal to the 200 . The very reliable values of the pressure distribution coefficients, the coefficients of friction and buoyancy, and the Strouhal number were found. Richardson et al. [10] investigated the flow around the bridge pier without and with the scour hole. Also, they made a comparison with experimental result. They discover that the flow patterns around a pier of the bridge are well simulated by Flow3D hydrodynamic model. Chang el al. [11] adopted the model of large eddy simulation to find the solution of the flow equations around the pier of the bridge with a fixed bed and no scour.

The hydraulic functionality of the vane nearby a cylinder, concentrated on create a hydraulic field around the vane. Here, when the flow passes the vane and becomes ready to enter the upstream region of the cylinder, the turbulence at the upstream of the cylinder is not strong as compared with turbulence at the vane upstream region. Because of the vane destroy strongly and dissipate the flow energy and this occurs suddenly when the flow encounters the vane. Also, the flow separation will rise owing to the presence of the vane. The presence of the vane at the upstream of the cylinder, play an important role in civil engineering application like the presence of the vane at the upstream of the bridge pier will reduce the scour depth around the pier at high efficiency and this will lead to increase the serviceability life of the pier and avoid the hydraulic failure owing to the scour around the pier. The target of the present paper concentrates on the investigation the 
influence of vane configuration and direction on the neighboring cylinder. Here the vane is placed at the upstream of the cylinder. This subject deal with challenge problem happens between the vane and the cylinder under the turbulent flow condition. This challenge emerged from the interference which happens between flow and vane in addition to vane and flow field around the cylinder. This interference reveals a noticeable situation with appear clear due to the existence of the vane.

\section{Numerical modeling and solution}

The current paper deals with three different cases. The first case is a cylinder without vane, while in the second and third case respectively the cylinder is nearby the vane. The modeling and simulation are performed in two-dimensions (2D) by utilizing the software ANSYS (fluent). Based on the geometry which is generated by ANSYS, fine meshes range from 29900 to 29993 elements. The numerical analysis depends on finite volume method. Figure 1 illustrate the three different cases, Figure 1 contains all the following dimensions cylinder diameter, vane length and width, also domain length and width. Figure 2 illustrate the computational grids for the three cases which used in this paper. The assumption which are adopted in the modeling and simulation of the current problem are listed below:

1- The flow material is water.

2- Water is considered as incompressible material.

3- The physical properties of water are considered constant.

Table 1 shows the water properties. Table 2 reviews the boundary condition of the hydraulic system. The channel bed described as horizontal, flat and fixed, while the walls is considered vertical. Both the vane and a cylinder are considered submerged. The adopted velocity of water is equal to the $0.1 \mathrm{~m} / \mathrm{sec}$ and the time of analysis equal to 8 second.

Table 1. Water Properties $[12,13]$.

\begin{tabular}{ll}
\hline Density ( ) & Viscosity $(\mathrm{kg} / \mathrm{m} . \mathrm{s})$ \\
998.2 & 0.001003 \\
\hline
\end{tabular}

Table 2. Boundary Condition of the Hydraulic System. The numerical analysis deals with the studying of

\begin{tabular}{ll}
\hline Inlet & Velocity at inlet \\
\hline Outlet & Pressure at outlet \\
Cylinder & No slip-Wall
\end{tabular}




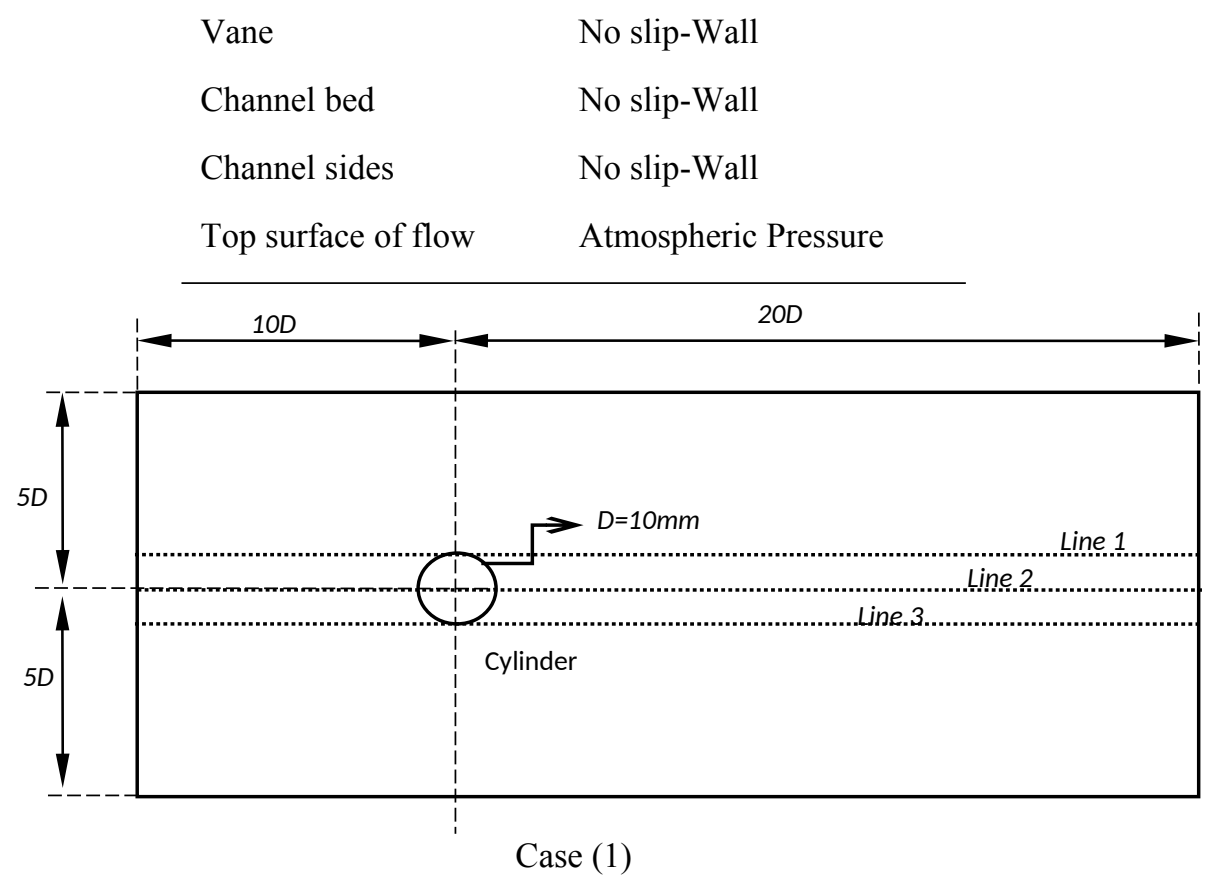

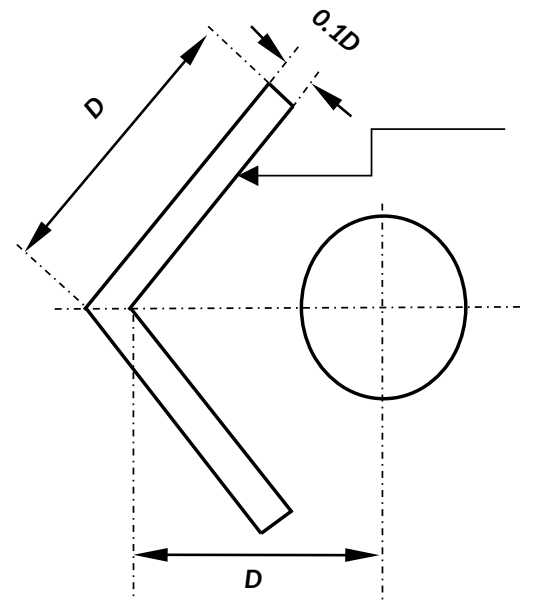

Case (2)

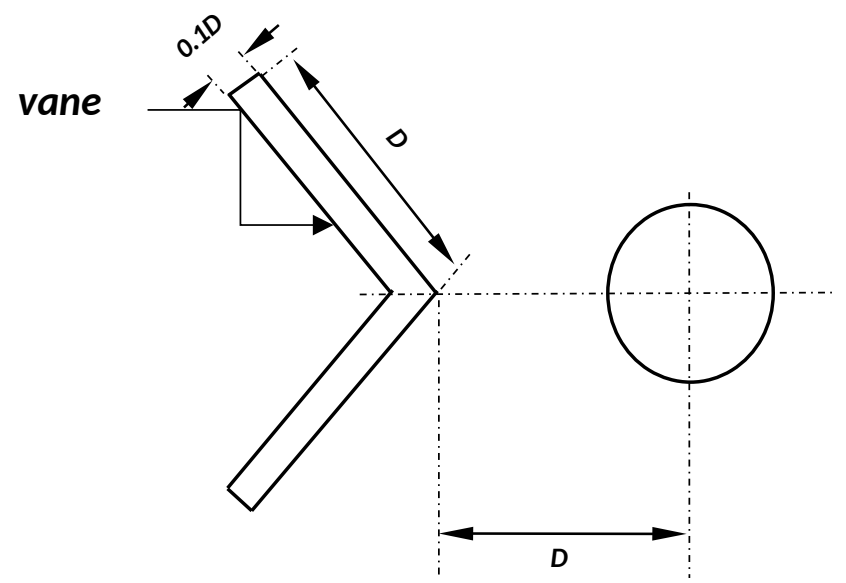

Case (3)

Figure 1. Shows the configuration and direction of vane neighboring to a cylinder, also shows a cylinder alone. 


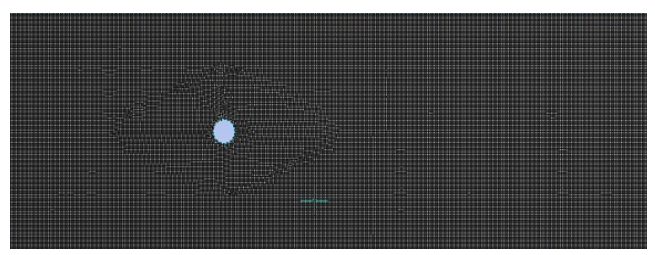

Case (1) with 29993 elements

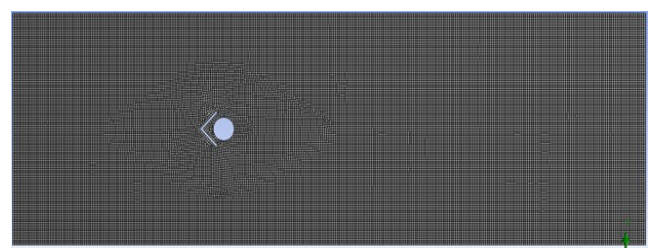

Case (2) with 29926 elements

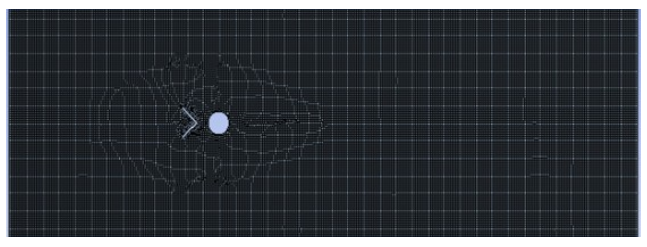

Case (3) with 29900 elements

Figure 2. Shows the mesh of the different cases.

The CFD governing equations that will be used for the simulation are the conservation of momentum equation and incompressible fluid continuity equation is shown in equations (1) to (4), respectively.

Verification

It is very important to undergo verification in the software before adopting it in solving the present problem. So, the fluent software is used for analysis of the case study in reference [12] and the results which have been obtained from the analysis are compared with the previous results that have been obtained in the reference [12]. Case studied in reference [12] concentrated on the flow field around a square. Three-dimensional analysis is used in the simulation of the reference [12] case studied. Table 3 reviews the information of reference [12] case studied, while Table 4 shows the obtained value of the drag coefficient from the study in reference [12] and the drag coefficient value from the current study.

Table 3. Case study information

\begin{tabular}{lllll}
\hline $\mathrm{D}(\mathrm{m})$ & $\mathrm{V}(\mathrm{m} / \mathrm{s})$ & $\mathrm{H}(\mathrm{m})$ & & $\operatorname{Re}$ (no unit) \\
1 & 0.292 & 2 & 1.225 & 20000 \\
\hline
\end{tabular}

Table 4. Comparison between the previous study [12] and current problem. 


\begin{tabular}{llll}
\hline Reference studies & Reynolds Number & Setup & \\
\hline Zaid et al. [12] & 20000 & Numerical & 2.1951 \\
Present study & 20000 & Numerical & 2.15 \\
\hline
\end{tabular}

\section{Result and Discussion}

The simulation of the current problem is done by the finite volume method and is explained briefly in this section.

Strain Rate: Figure 3 concentrates on the contour variation of strain rate for different case studies. Generally, strain rate refers to the gradient of the flow velocity. The velocity gradient increases from case to case. It is obvious from figure, case (2) has high strain rate as compared with the cases (1) and (3). for case (2) high separation of water will happen at the vane front and this separation makes a reduction in water flow velocity before it passes the cylinder and this reduction continues with increase in distance, therefore, the gradient of flow velocity increasing at the cylinder downstream. In case (2) the obstruction which is provided by the vane play an important role in reducing the flow velocity and increases the strain rate or flow velocity gradient, while case (3) has less effect as compared with case (2). In case (1) there is no obstruction which confines and dissipates water flow, therefore, there is no noticeable shortage in the value of flow velocity when the flow past the cylinder, so there is no significant alter in strain rate at the downstream region of the cylinder. Figure 4 shows the variation in strain rate in the direction of the flow, the Figure 4 adopts three lines in longitudinal direction one of the lines pass at the center of the cylinder while the remainder pass at the edge of the cylinder. Figure shows for all cases, that the maximum value of the strain rate always occurs in case (2) at each line which passes at the center and the edge of the cylinder. While the strain rate in case (1) and case (3) has moderate values. For all cases when the flow passes the cylinder the strain rate vanishes.

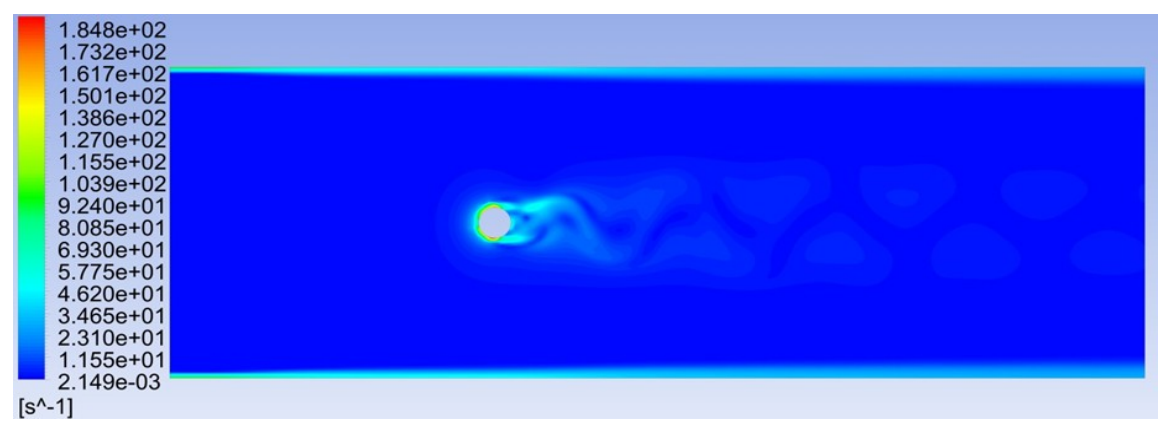

Case (1) 


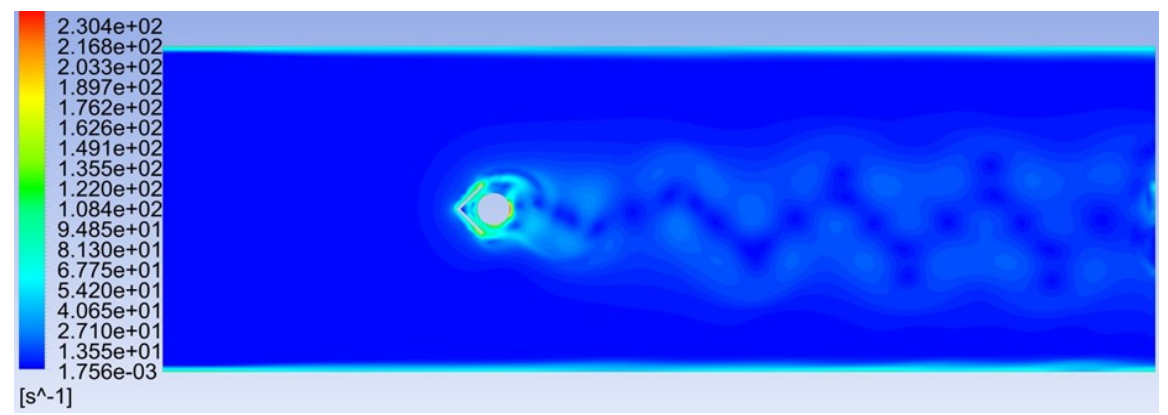

Case (2)

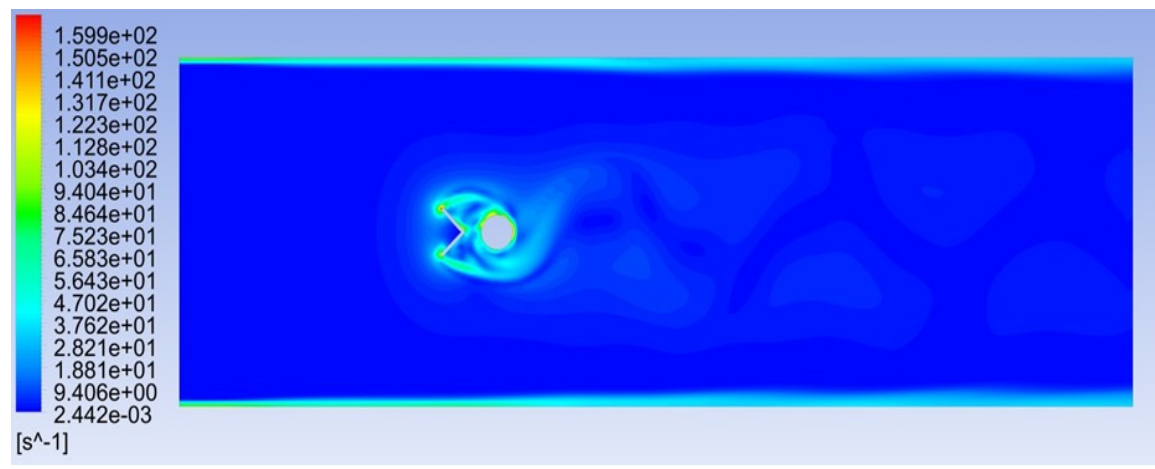

Case (3)

Figure 3. Shows the change in strain rate for different cases.

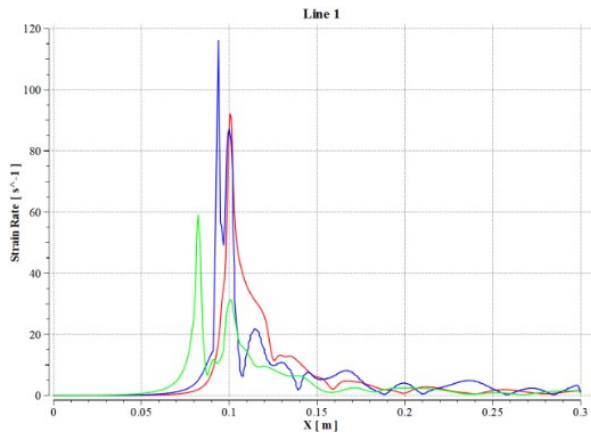

(a)

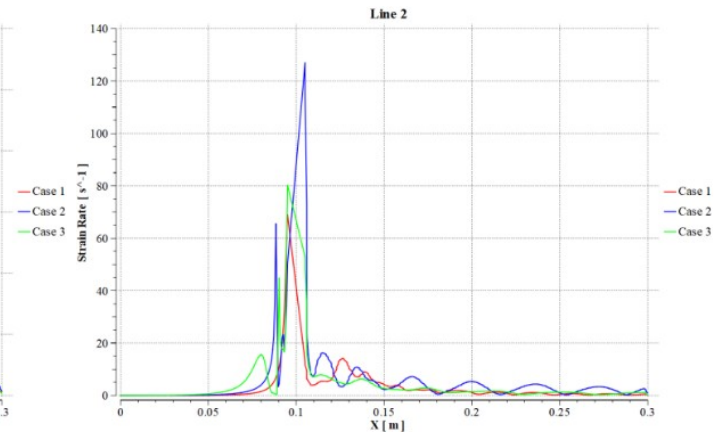

(b) 


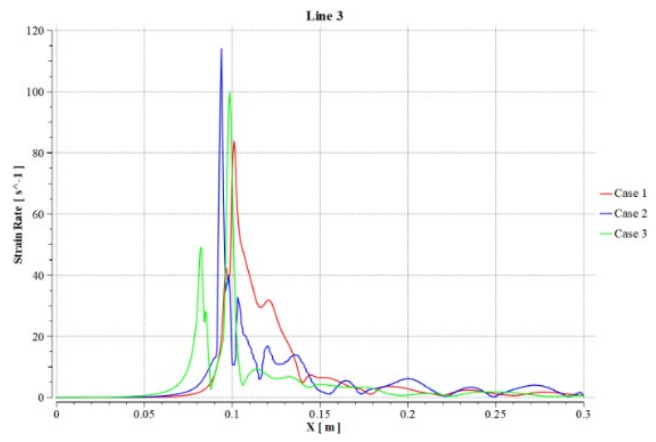

(c)

Figure 4. Shows the change in strain rate for different line in the direction of the flow (a) Line 1, (b) Line 2 and (c) Line 3.

Figure 5 concentrates on the contour variation of stream function for different case studies. The stream function is used to describe the stream line which generates around the body like the cylinder and the vane. When the cylinder is alone case (1), the stream line which is generated at the cylinder downstream has small amplitude. While in case (2) the effect of the vane on the stream line appears clearly. Here, the vane separate, dissipate and work to deflect the flow direction, therefore, all these processes will reflect on the shape of the stream line around the cylinder, while case (3) has moderate hydraulic response between case (1) and case (2). Generally, the change in stream line depends mainly on the flow velocity and volumetric flow. In case (2) the water undergoes more deformation as compare with the other cases.

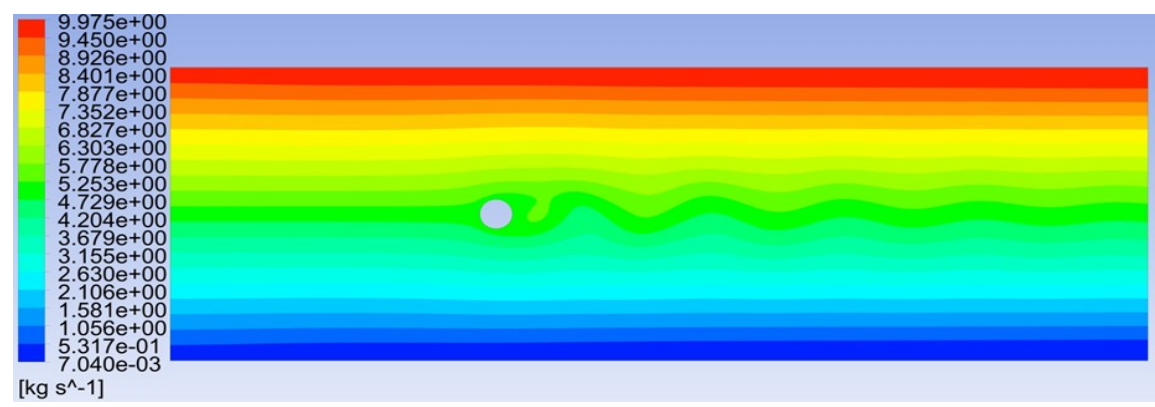

Case (1) 


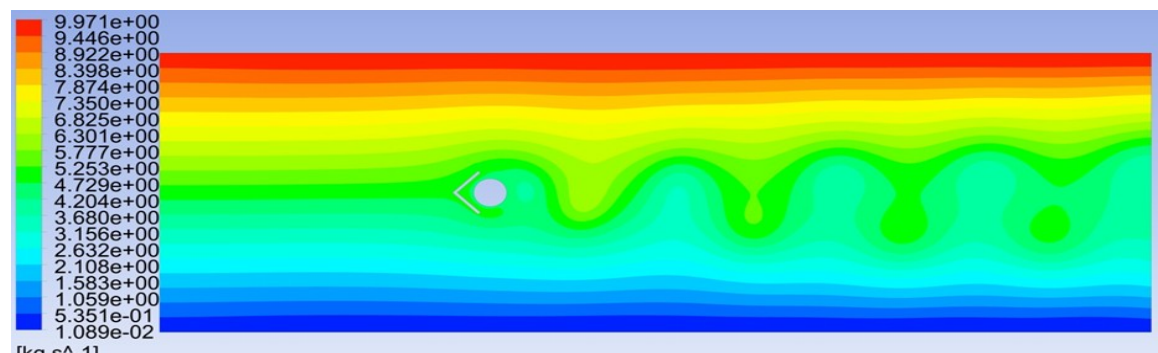

Case (2)

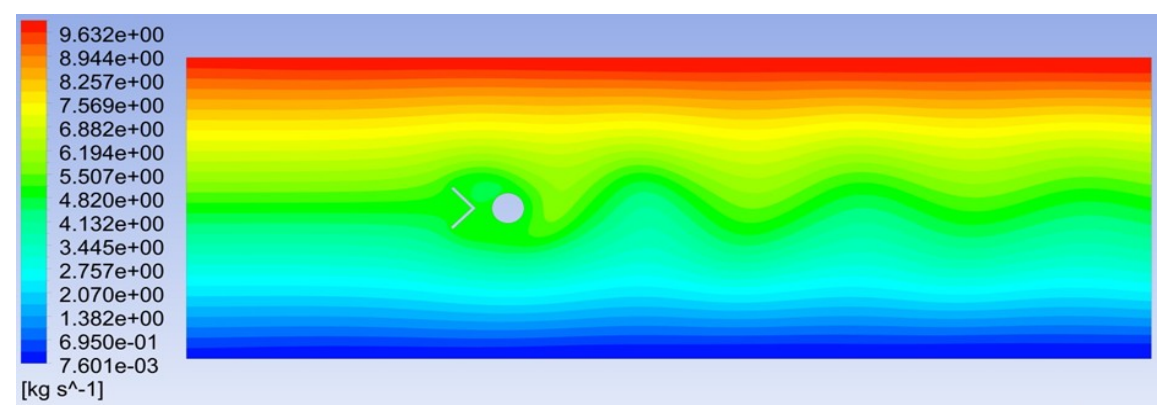

Case (3)

Figure 5. Shows the change in stream function for different cases.

Figure 6 concentrate on the contour variation of swirling for different case studies. Generally swirling refers to the eddy which generates near the obstacle. Here, the obstacle is the cylinder and the vane. For all cause, it is obvious normal swirling (eddy) and this means that the turbulence which create the eddy is not high. In this figure the swirling is normal to the xy-plane because the problem is analysis in two dimensions.

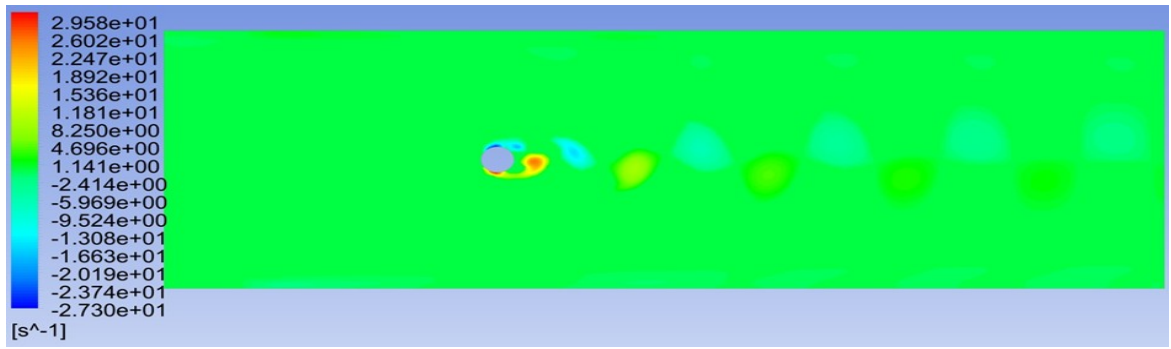

Case (1) 


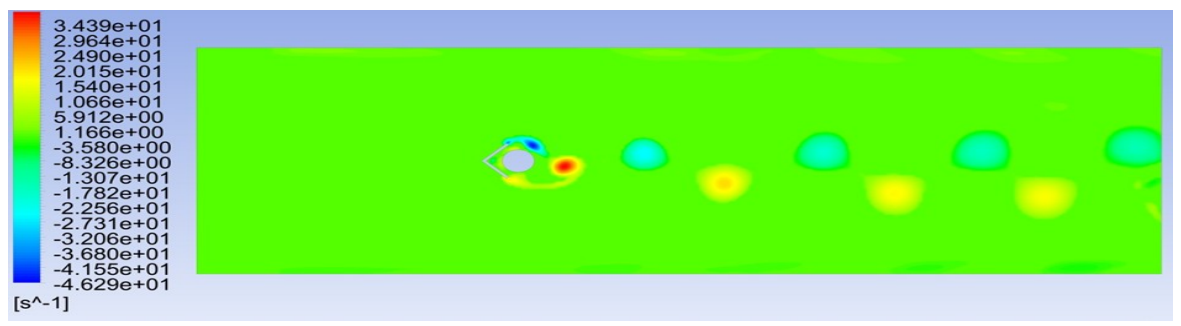

Case (2)

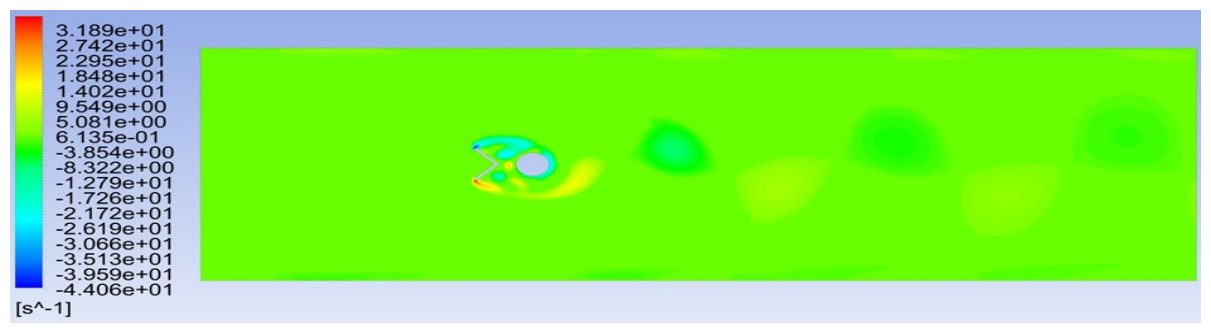

Case (3)

Figure 6. Shows the change in swirling normal to $\mathrm{z}$.

Figure 7 illustrate the flow velocity contour in the direction of the flow for all cases. We observe a strong eddy occur in case (2) when the cylinder is near by the vane as compare with case (1) and case (2). Also, at the near lateral side of the cylinder in case (1), we observed a strong oscillatory flow representing by the two vortexes at the side of the cylinder. For cases 2 and 3 the oscillatory will develop at the downstream of the cylinder. The oscillatory flow at the downstream of case (2) can be described as stronger as compared with other cases.

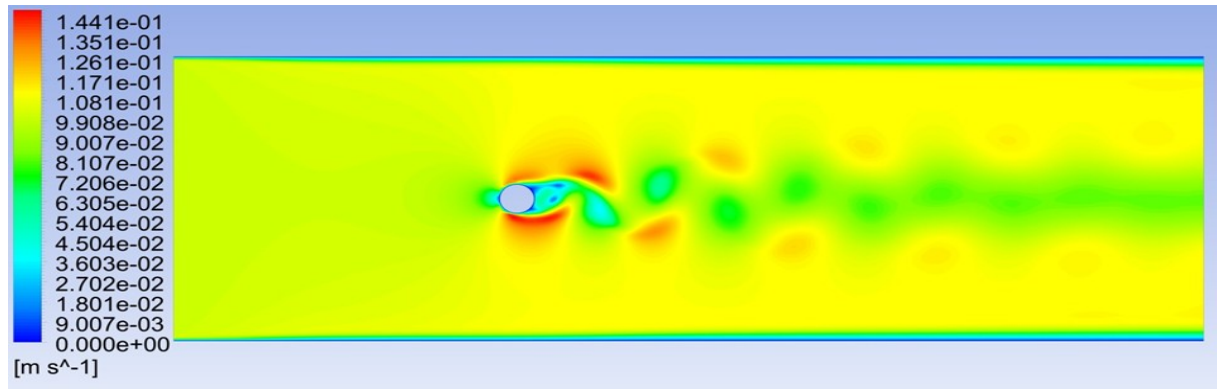

Case (1) 


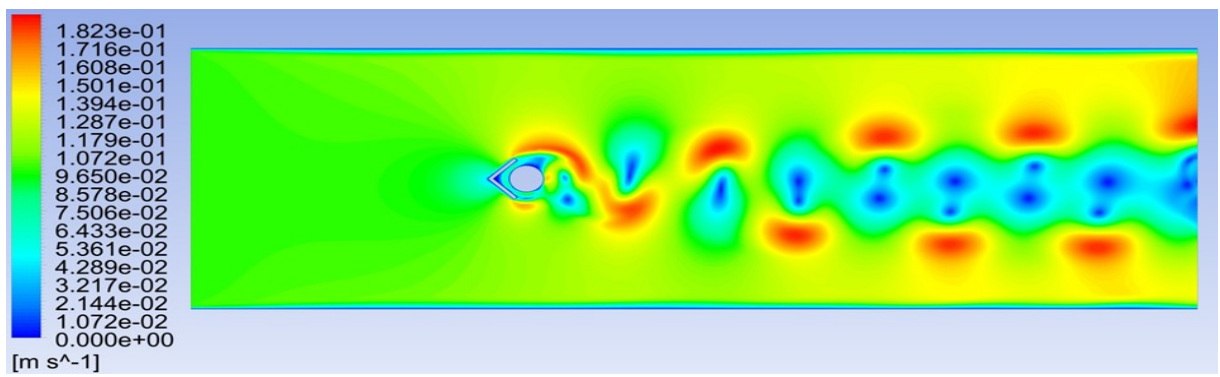

Case (2)

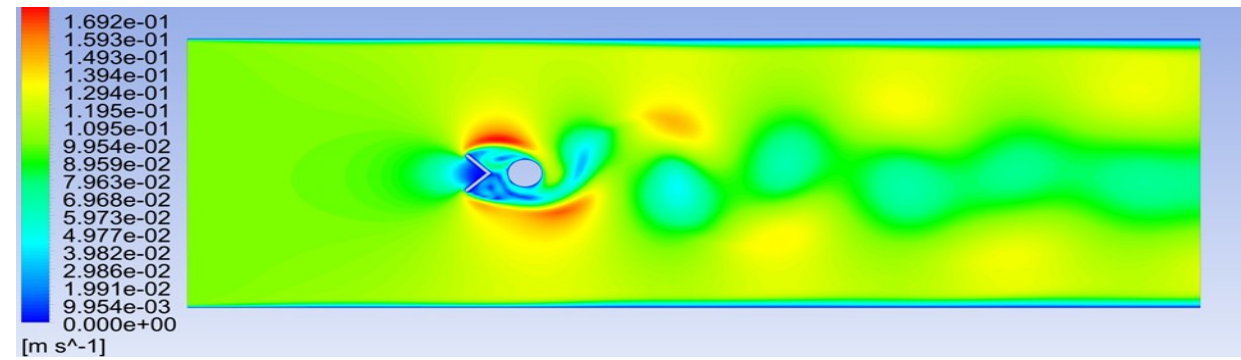

Case (3)

Figure 7. Shows the counter of flow velocity magnitude.

Figure 8 illustrate the variation in the radial flow velocity for different cases. It is evident from the figure when the flow passes the cylinder (case -1) or the cylinder which is nearby the vane (case -2 and case- 3 ) the turbulence and the eddy grow and develop gradually, regardless of especially the direction and the configuration. Dramatic alter happens in radial velocity relies on the points of the fluid separation, turbulent dissipation and the flow velocity gradient with distance. The radial flow velocity at the downstream of case (2) can be described as more alter as compared with other cases.

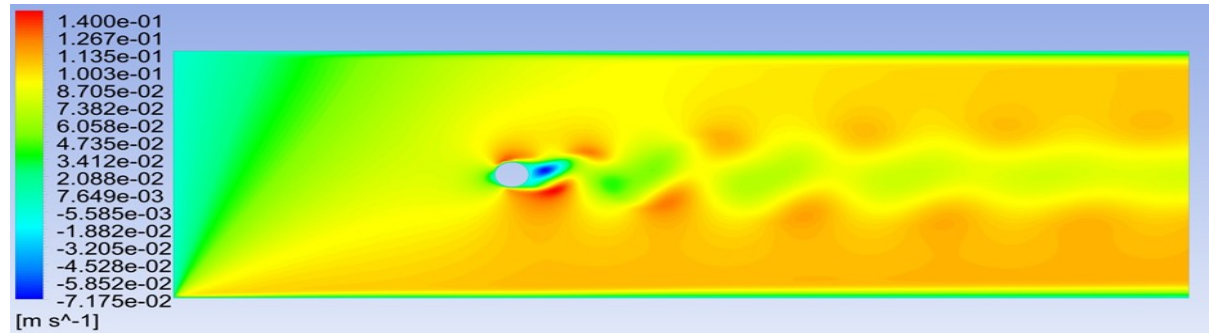

Case (1) 


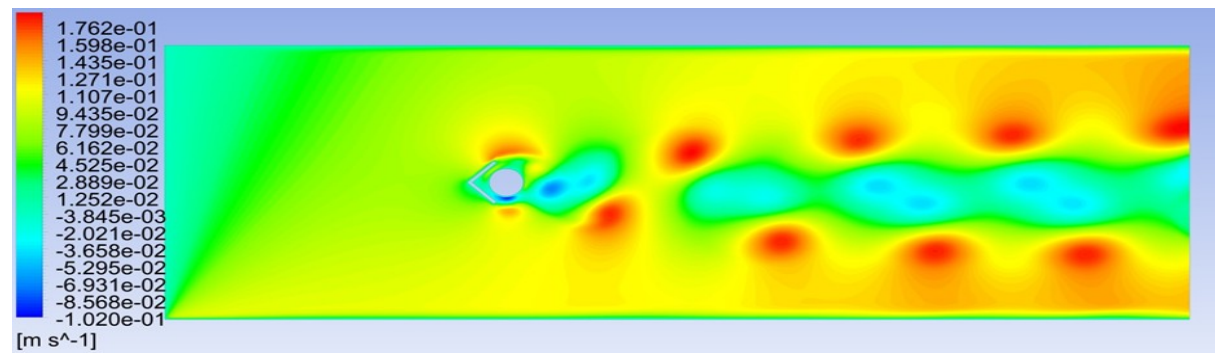

Case (2)

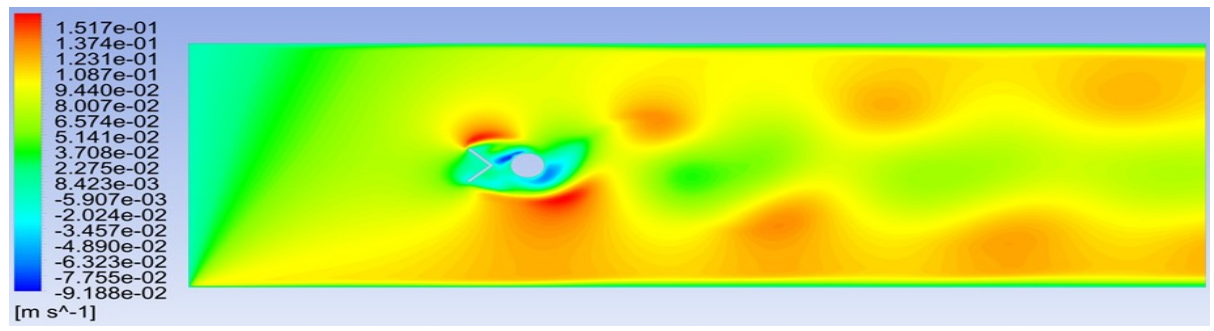

Case (3)

Figure 8. Shows the counter of radial flow velocity magnitude.

Figure 9 illustrates the vorticity contours map around a cylinder without vane and with vane respectively. Here, the mode of the vortex formation depends on the pressure around the cylinder or the pressure around the vane. In case (1) thick layers are composed mostly of vorticity of small magnitude around the cylinder surface, while in cases (2 and 3$)$ the thick layers will be created around the vane instead of the cylinder surface. For all cases the vorticity will develop strongly at the downstream of the cylinder especially in case (2) as compared with other cases. The projection area of the cylinder and the vane, also the location of the flow separation point represent the major factors which control the vorticity. Figure 10 illustrate the pressure contour variation around alone cylinder (case-1) and around cylinder which nearby the vane respectively. The pressure values change form positive at upstream region to the negative values at the downstream region with significant fluctuation depends on the case study. Generally, for all cases, the contour refers to the maximum and positive values of pressure will be happened at the upstream regardless the existence of the vane, while the minimum and negative values will be dominate at the downstream regardless the existence of the vane. The existence of the vane leads to the variation in pressure values and makes a difference between the upstream values and downstream values. 


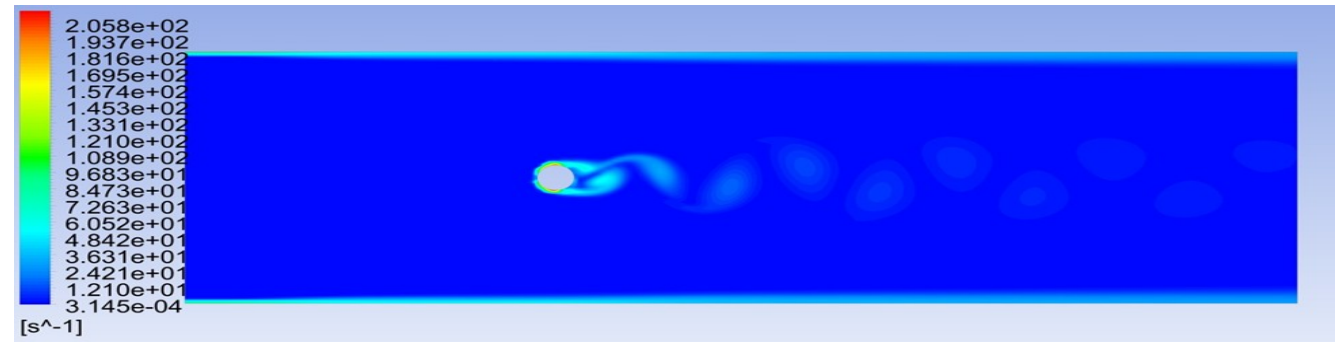

Case (1)

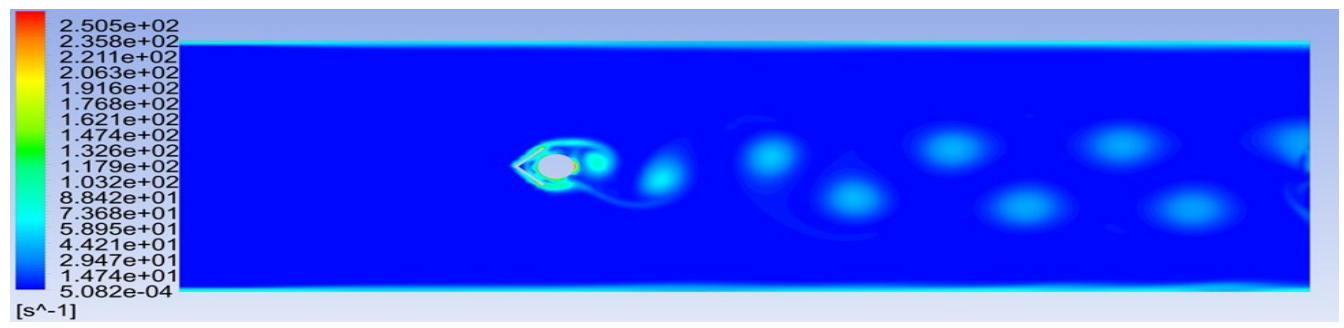

Case (2)

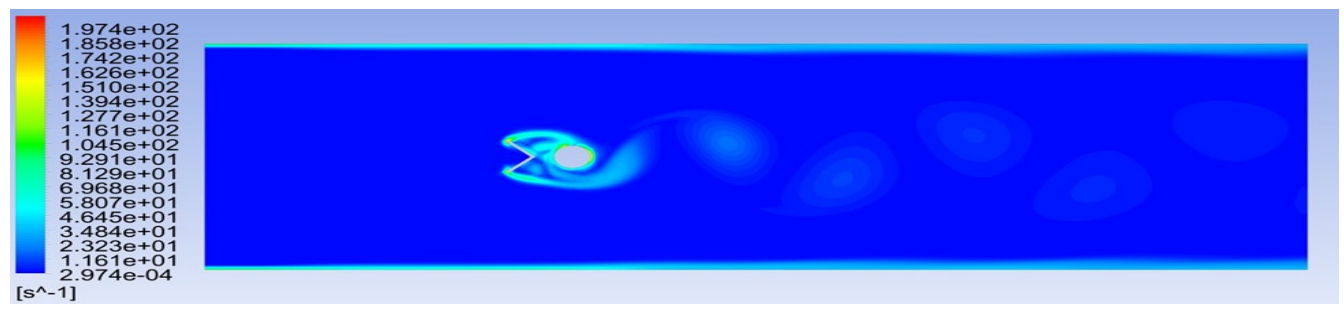

Case (3)

Figure 9. Shows the contour of vorticity

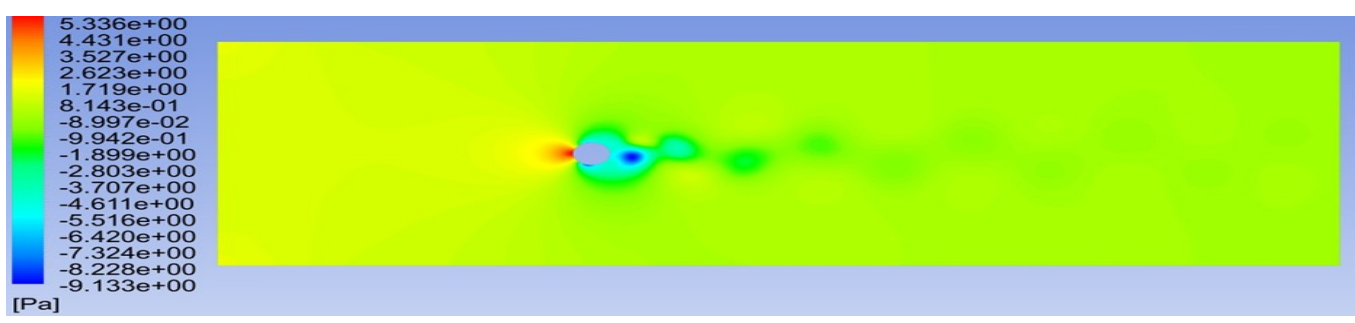

Case (1) 


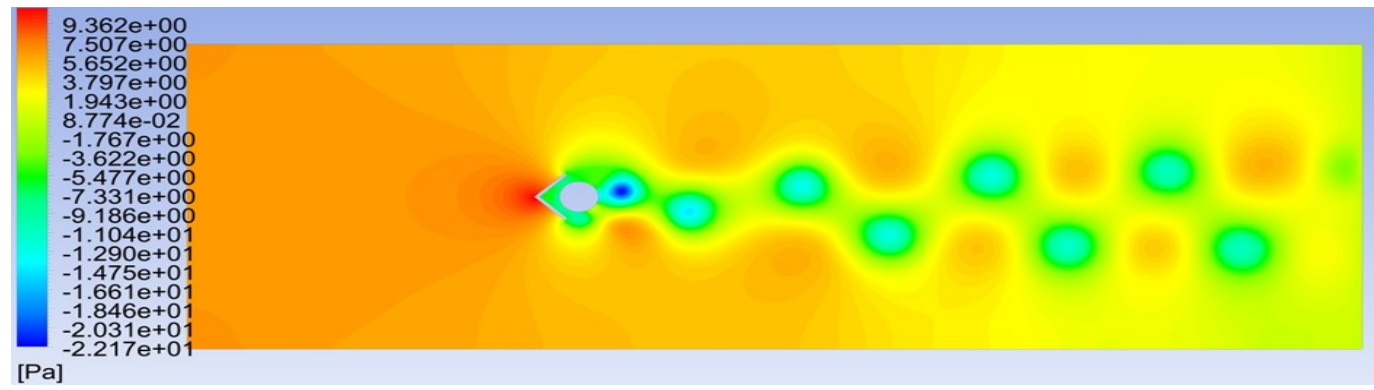

Case (2)

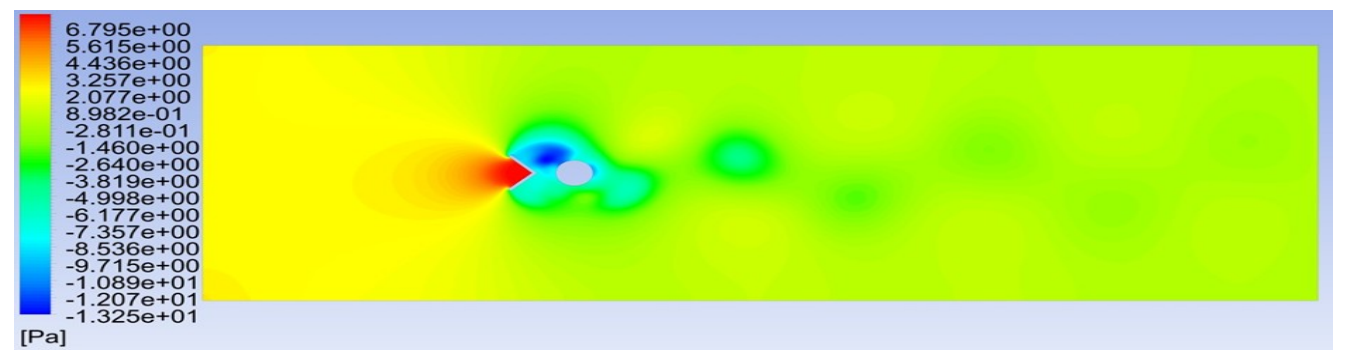

Case (3)

Figure 10. Shows the pressure contour variation.

Figure 11 reviews the pressure coefficient contour variation. The pressure coefficient values change from positive at upstream to the negative at the downstream. Regardless of the vane's presence, this happen owing to the influence of water flow separation and water velocity losses which happen when the flow past the vane.

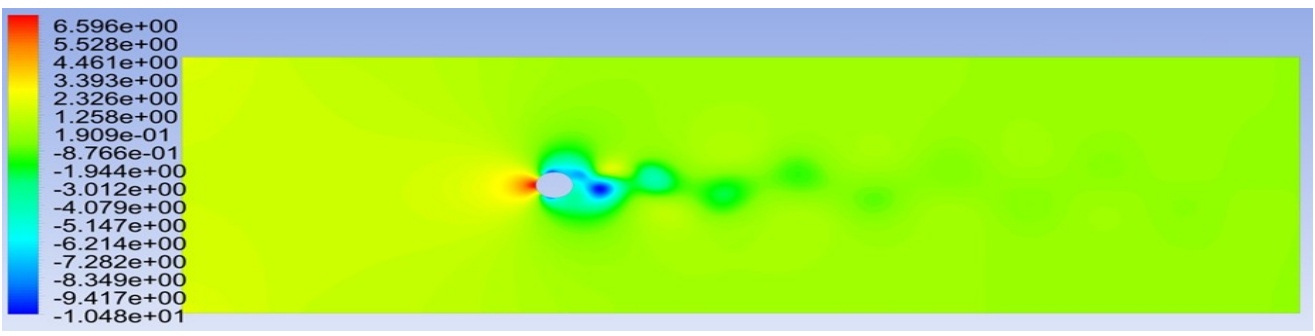

Case (1) 


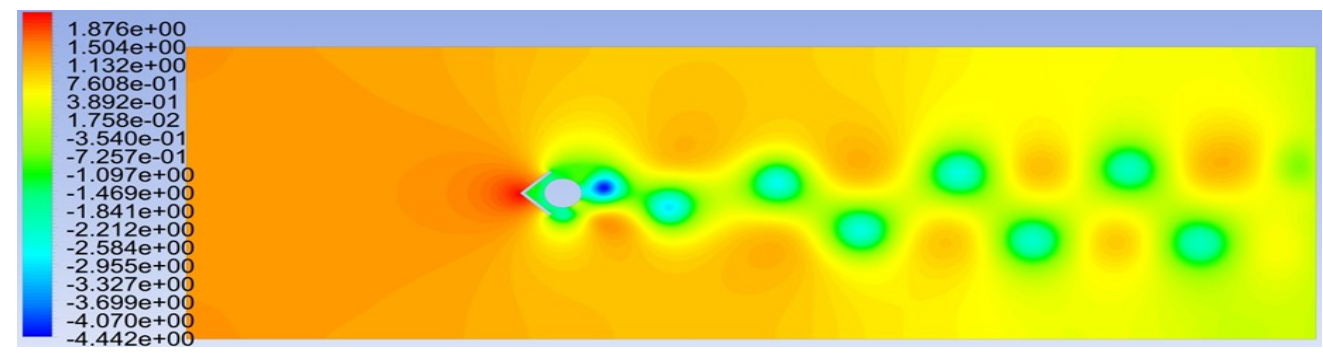

Case (2)

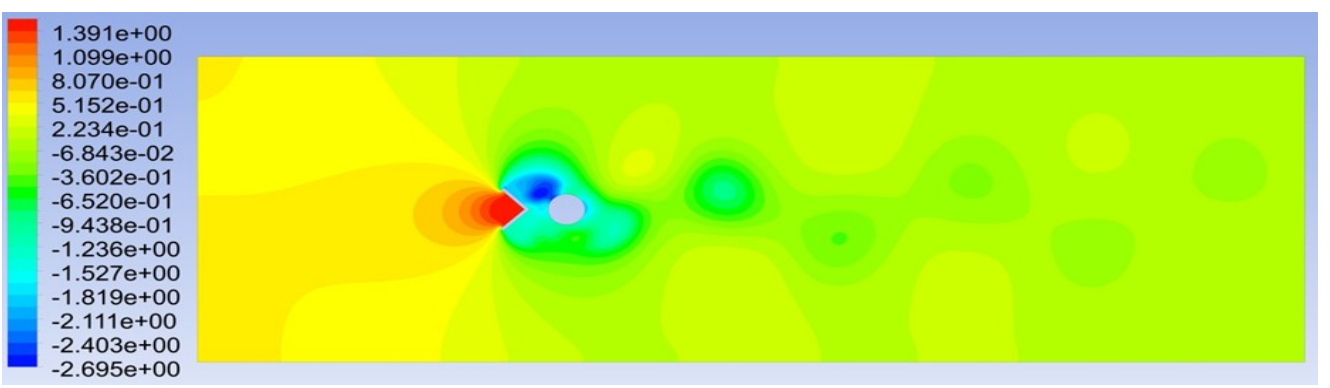

Case (3)

Figure 11. Shows the pressure coefficient contour variation.

Figure 12 shows the variation in the pressure, Figure 12 adopts three lines in longitudinal direction one of the lines pass at the center of the cylinder while the remainder pass at the edge of the cylinder. Figure shows for all cases, case (3) always represents the upper limit in positive and case (2) always represent the lower limit in negative values respectively, while case (1) always represents the moderate limit in positive and negative values respectively. It is clear from the figure that the pressure always positive at the upstream of the cylinder and the pressure always negative at the downstream of the cylinder. The variation in pressure occurs owing to high flow separation by the vane and the cylinder, also the dissipation in flow velocity will reflect on the pressure distribution. 


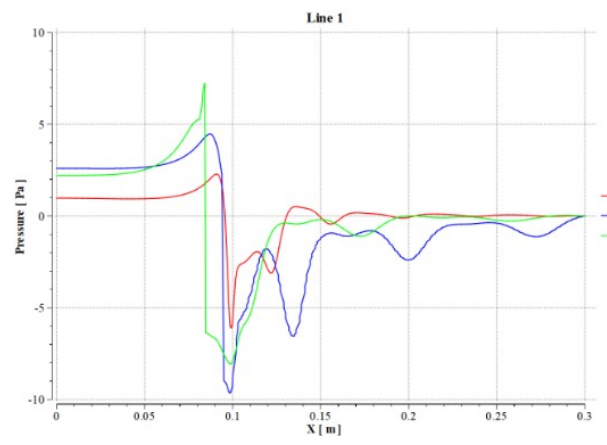

(a)

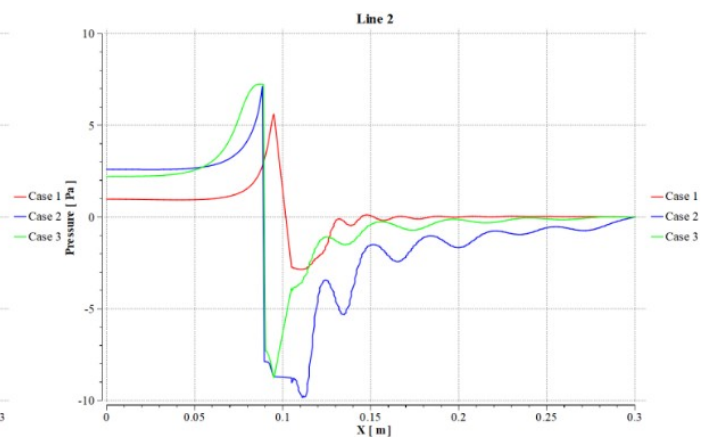

(b)

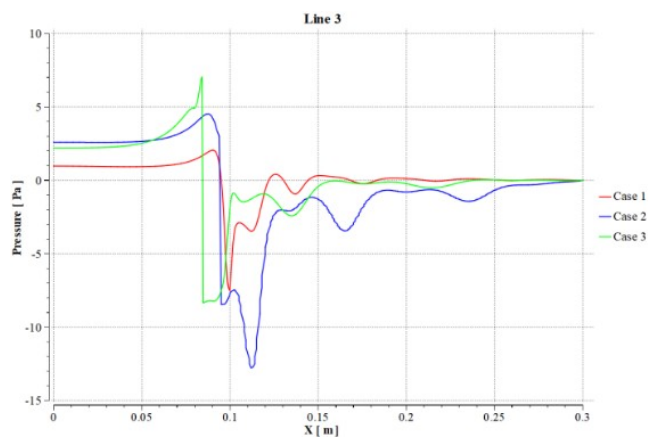

(c)

Figure 12. Shows the pressure variation at different lines (a) Line 1, (b) Line 2 and (c) Line 3.

Figure 13 shows the variation in drag coefficient with time, generally for all cases the drag coefficient has periodic response. The presence of vane neighboring to the cylinder reduce the drag coefficient as compared with alone cylinder regardless the direction and configuration of the vane, because of the separation zone occur at the vane instead of the cylinder this will reduce the vortex separation from the cylinder surface. Negative drag coefficient on a cylinder appears to be counter-intuitive in spite of there is no principle or law that prohibits it. The pressure variation plays a vital role in produce the negative drag. 


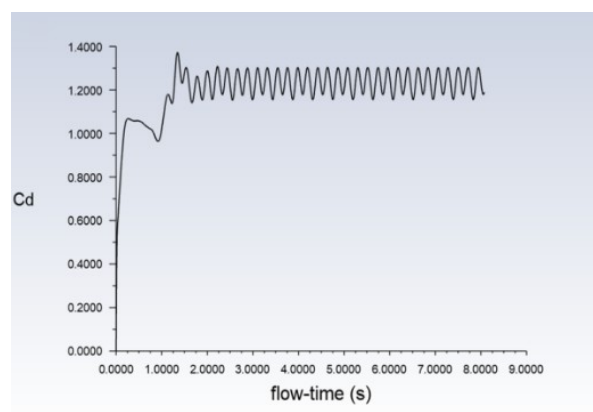

Case (1) $\mathrm{Cd}=1.1899269$

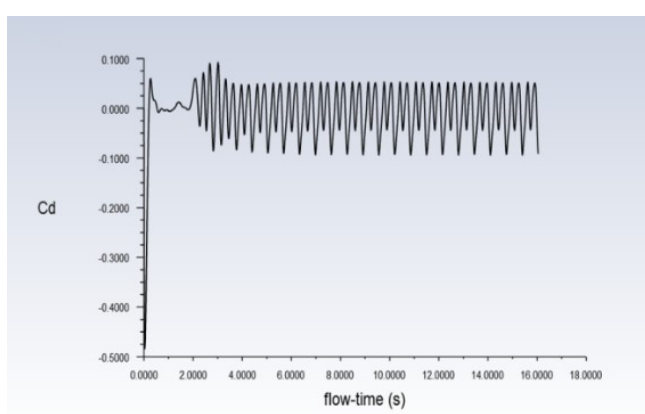

Case (2) $\mathrm{Cd}=-0.091996409$

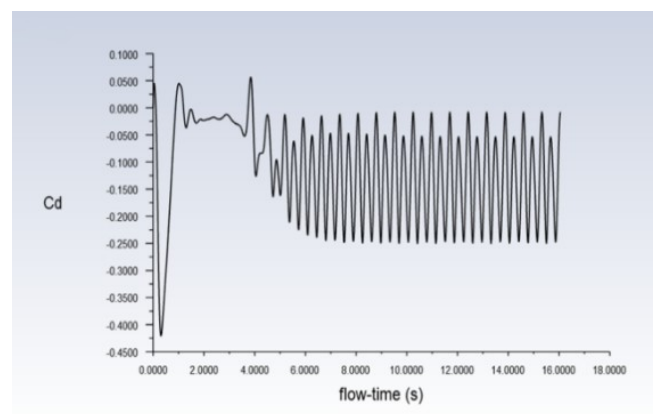

Case (3) $\mathrm{Cd}=-0.0093440274$

Figure 13. Show the variation of drag coefficient with time.

\section{Conclusion}

Flow field around cylinder without vane and with vane had been studied in this paper. Studying covers the influence of strain rate, stream function, flow velocity, radial velocity, swirling, vorticity, pressure, pressure coefficient and drag coefficient. From this study it is found that the vane direction and configuration have a major impact on the flow field around a cylinder. It is found that the vane has significantly affect in reduce the drag coefficient to the reasonable and satisfactory value as compare with drag coefficient without vane. It is found that the pressure distribution and pressure coefficient are more sensitive to the presence of the vane at the upstream of the cylinder. The flow velocity gradient controls the strain rate while the stream function expresses how much the flow suffer deformation when it passes the obstructions. The velocity field has allowed the showing of the eddy zone, vortex zone and recirculation zone. The vorticity is affected by the pressure and flow separation zone. The response of flow field around the cylinder is more sensitive to the existence of the vane. 


\section{References}

[1] Sowoud, K.M., AL-Filfily, A.A., Abed, B.H., "Numerical Investigation of 2D Turbulent Flow past a Circular Cylinder at Lower Subcritical Reynolds Number". IOP Conf. Series: Materials Science and Engineering 881 (2020) 012160, doi:10.1088/1757-899X/881/1/012160.

[2] Mojtaba D, 2016 "Numerical Investigation of the Fluid Flow around and Pas a Circular Cylinder by ANSYS Simulation" International Journal of Advanced Science and Technology 92 49- 58.

[3] Bhagat, K. C., Soren, S. K. and Chaudhary, S. K., 2016 "Experimental and Numerical Analysis of Different Aerodynamic Properties of Circular Cylinder". International Research Journal of Engineering and Technology (IRJET) 3(9) 1112-17.

[4] Yuce, M.I., Kareem, D.A., A "Numerical Analysis of Fluid Flow around Circular and Square Cylinders" Journal American Water Works Association, http://dx.doi.org/10.5942/ jawwa.2016.108.0141, (E546- E554).

[5] Zhao Wei, Huhe Aode, "Large-Eddy Simulation of Three-Dimensional Turbulent Flow around a Circular Pier" Journal of Hydrodynamics. Ser. B, 2006,18(6):765-772.

[6] Davis, R.W., Moore, E. F., Purtell, L.P. "A numerical-experimental study of confined flow around rectangular cylinders" Phys Fluids 1984;27(1):46-59.

[7] B.W.V. Oudheusden, F. Scarano, N.P.V. Hinsberg, D.W. Watt "Phase-Resolved Characterization of Vortex Shedding in the Near Wake of a Square Section Cylinder at Incidence" Experiments in Fluids, Vol. 39, pp. 86-98, 2005. https://doi.org/10.1007/s00348-005-0985-5_

[8] B. Gera, P.K. Sharma, R.K. Singh "CFD Analysis of 2D Unsteady Flow Around a Square Cylinder" International Journal of Applied Engineering Research, Vol. 1, No. 3, pp. 602- 610, 2010 .

[9] H. Bai, J.W. Li" Numerical Simulation of Flow Over a Circular Cylinder at Low Reynolds Number" Advanced Material Research, Vols. 255-260, pp. 942-946, 2011. https://doi.org/10.4028/www.scientific.net/AMR.255-260.942.

[10] Richardson, J.S., Pancheng V.G. "Three dimensional simulation of scour inducing flow at bridge piers" J.Hydrau. Eng. ASCE 1998:124(5)

[11] Chang, WY., Lai JS, Yen CI "Simulation of scour depth evolution at pier nose" In: Proceedings of the 1999 international water resources publications, LLC, Highlands Ranch, CO.:1999. 
[12] Zaid M., Yazdanfar Z., Chowdhury H., Alam F."Numerical modeling of flow around a pier mounted in a flat and fixed bed" Energy Procedia, 160: 51-59, 2019. 